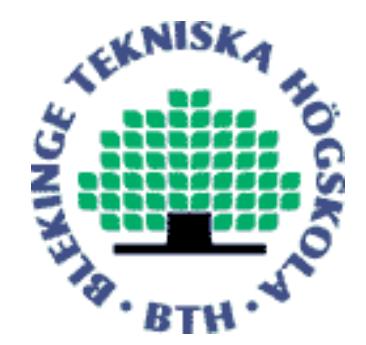

Copyright @ 2014 IEEE.

Citation for the published paper:

Title:

In press: A Health-IoT Platform Based on the Integration of Intelligent Packaging, Unobtrusive Bio-Sensor and Intelligent Medicine Box

Author:

Geng Yang, Xie Li, Matti Mäntysalo, Xiaolin Zhou, Zhibo Pang, Li Da Xu, Sharon Kao-Walter, Qiang Chen, Lirong Zheng

Journal:

IEEE Transactions on Industrial Informatics

Year:

2014

Vol:

Issue:

Pagination:

URL/DOI to the paper:

10.1109/TII.2014.2307795

This material is posted here with permission of the IEEE. Such permission of the IEEE does not in any way imply IEEE endorsement of any of BTH's products or services Internal or personal use of this material is permitted. However, permission to reprint/republish this material for advertising or promotional purposes or for creating new collective works for resale or redistribution must be obtained from the IEEE by sending a blank email message to pubs-permissions@ieee.org.

By choosing to view this document, you agree to all provisions of the copyright laws protecting it. 


\title{
A Health-IoT Platform Based on the Integration of Intelligent Packaging, Unobtrusive Bio-Sensor and Intelligent Medicine Box
}

\author{
Geng Yang, Li Xie, Matti Mäntysalo, Xiaolin Zhou, Member, IEEE, Zhibo Pang, Li Da Xu, Sharon \\ Kao-Walter, Qiang Chen, Lirong Zheng, Senior Member, IEEE
}

\begin{abstract}
In-home healthcare services based on the Internet-of-Things (IoT) have great business potential; however, a comprehensive platform is still missing. In this paper, an intelligent home-based platform, the iHome Health-IoT, is proposed and implemented. In particular, the platform involves 1) an open-platform-based intelligent medicine box (iMedBox) with enhanced connectivity and interchangeability for the integration of devices and services, 2) intelligent pharmaceutical packaging (iMedPack) with communication capability enabled by passive radio-frequency identification (RFID) and actuation capability enabled by functional materials, and 3 ) flexible and wearable bio-medical sensor device (Bio-Patch) enabled by the state-of-the-art inkjet printing technology and system-on-chip. The proposed platform seamlessly fuses IoT devices (e.g., wearable sensors, intelligent medicine packages, etc.) with in-home healthcare services (e.g., telemedicine) for an improved user experience and service efficiency. The feasibility of the implemented iHome Health-IoT platform has been proven in field trials.
\end{abstract}

Manuscript received September 30, 2013; revised December 17, 2013 accepted February 9, 2014. Date of publication xxx; date of current version xxx. This work is financially supported by Vinnova (The Swedish Governmental Agency for Innovation Systems) through the VINN Excellence centers program. This work is supported in part by the National Natural Science Foundation of China (NNSFC) under Grant 71132008, and U.S National Science Foundation under Grant SES-1318470 and 1044845. M. Mäntysalo is supported by Academy of Finland under Grant 251882.

G. Yang, L. Xie, and Q. Chen are with the iPack Vinn Excellence Center, School of Information and Communication Technology, Royal Institute of Technology (KTH), Stockholm, Sweden (e-mail: gengy@kth.se; lixi@kth.se; qiangch@kth.se).

M. Mäntysalo is with Department of Electronics, Tampere University of Technology. FI 33101 Tampere, Finland (e-mail: matti.mantysalo@tut.fi).

X. L. Zhou is with Department of Communication Science and Engineering, Fudan University, Shanghai, China (e-mail: zhouxiaolin@fudan.edu.cn).

Z. Pang is with $\mathrm{ABB} \mathrm{AB}$, Corporate Research, Västerås, Västmanland, Sweden (e-mail: pang.zhibo@se.abb.com).

L. D. Xu is with the Institute of Computing Technology, Chinese Academy of Sciences; Shanghai Jiao Tong University; University of Science and Technology of China, and Old Dominion University, Norfolk, USA.

S. Kao-Walter is with School of Engineering, Blekinge Institute of Technology (BTH), Karlskrona, Sweden (e-mail: sharon.kao-walter@bth.se).

L.-R. Zheng is with State Key Laboratory of ASICs and Systems, School of Information Science and Engineering, Fudan University, Shanghai, China, and also with the iPack Vinn Excellence Center, School of Information and Communication Technology, Royal Institute of Technology (KTH), Stockholm, Sweden (e-mail: lirong@kth.se).

Copyright (c) 2014 IEEE. Personal use of this material is permitted.
Index Terms-Internet-of-Things, Health-IoT, Bio-Patch, Intelligent Packaging, Intelligent Medicine Box, Printed Electronics.

\section{INTRODUCTION}

$\mathrm{N}$ OWADAYS, global ageing and the prevalence of chronic diseases have become a common concern [1]. Many countries are undergoing hospital restructuring by reducing the number of hospital beds and increasing the proportion of home healthcare [2]. A promising trend in healthcare is to move routine medical checks and other healthcare services from hospital (Hospital-Centric) to the home environment (Home-Centric) [2]. By doing so, firstly, the patients can get seamless healthcare at anytime in a comfortable home environment; secondly, society's financial burden could be greatly reduced by remote treatment; thirdly, limited hospital resources can be released for people in need of emergency care. In-home healthcare and services can drastically reduce the total expenditure on medical care or treatment. Therefore, it is urgent in the near future for the healthcare industry to develop advanced and practical health-related technologies and services by leveraging information and communication technology (ICT), and apply them directly in the home environment [3]. In order to track the physical status of the elderly and in the meanwhile keep them healthy, the following two daily tasks are essential: 1) real-time monitoring and analyzing vital signs to early-detect or predict life-threatening adverse events, 2) checking whether they are following their prescribed treatment, including taking their prescribed medicine on time. However, with rapidly aging populations, these daily tasks have brought great pressure and challenges to global healthcare systems. One review estimates that about $25 \%$ of the adult population do not adhere to their prescribed medication, which may lead to poor health outcomes and increased mortality [4]. Poor medication adherence is a major problem for both individuals and healthcare providers [4]. Technology improvements in healthcare facilities and services are highly desirable to meet the requirements of this giant group.

In the meantime, Internet-of-Things (IoT) has been recognized as a revolution in ICT since it started at the beginning of the 21 st century [5], [6]. IoT technology provides 


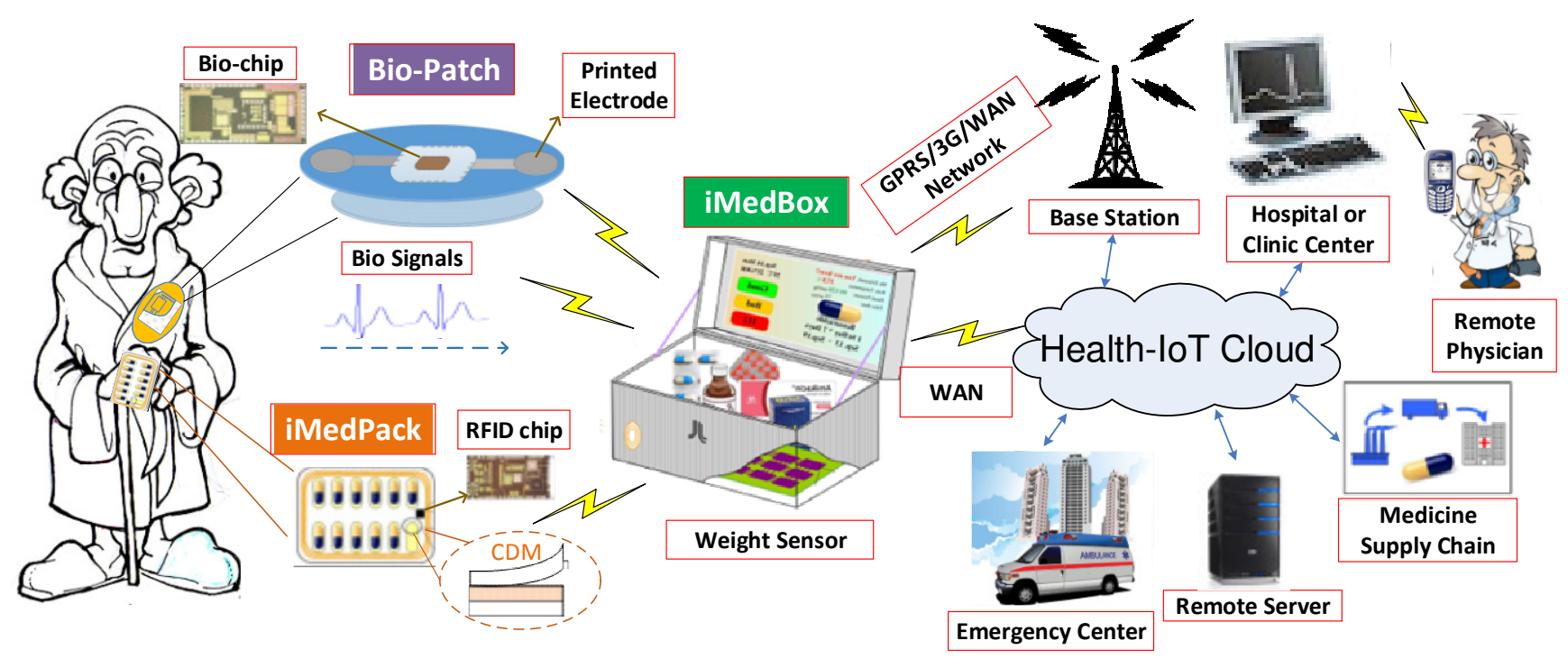

Fig. 1. Application scenario for the proposed iHome Health-IoT system.

the possibility to connect sensors, actuators or other devices to the Internet and is conceived as an enabling technology to realize the vision of a global infrastructure of networked physical objects [7]. IoT extends the Internet into our everyday lives by wirelessly connecting various smart objects [8], and will bring significant changes in the way we live and interact with smart devices [9], [10]. Various companies are exploring this domain as it can potentially unlock the door to new business opportunities.

As part of IoT - intelligent components, radio-frequency identification (RFID) tags [11], embedded sensors and actuators, etc. - have been rapidly developed and significantly expanded in scope. As a consequence, the number of IoT-based applications has boomed as well [12], [13]. All these technologies facilitate the deployment of IoT devices in the home environment for 24/7 healthcare [14]. Some researchers attempt to integrate wearable devices and systems in IoT scenario to achieve better e-health services [18], [19]. A mobile-phone based telemonitoring system for chronic disease management is presented in [20]. A smart health monitoring chair is introduced in [21] for non-invasive bio-signal measurement. However, these solutions are almost exclusively implemented using off-the-shelf components. As a result, the physical size, rigid nature and short battery life become limiting factors for potential long-term use.

Some research groups focus on the user-comfort issues, by leveraging advanced materials to develop user-friendly sensors. In [22]-[25], functional textiles are utilized to manufacture a sensorized garment for physiological monitoring, where electrocardiogram (ECG) signals are successfully recorded using fabric sensing elements. Although the integration of textile and electronics improves user comfort to some extent, one drawback lies in the manufacture process, where manual knitting and interconnection make the production costs relatively high.

Electronics on flexible substrates, e.g., plastic films, are quite promising for wearable sensors. Owing to their flexible nature, they provide a good fit on soft human bodies. In [26]-[28], an ultra-thin ( 2 um-thick) bionic skin with marvelous flexibility has been fabricated based on organic transistors. The artificial skin is embedded with temperature and tactile sensors to mimic the functions of the real skin. Ultimately, it will be possible to wrap the electronic skin around mechanical bones to build a prosthetic hand [29]. However, due to the process variation, mismatches may occur in the fabricated thin-film transistor [27]. As a consequence, the implemented amplifier array presents a degraded signal-to-noise ratio, leading to a noisy or even distorted output.

On the whole, the above-mentioned systems focus either on making improvements to a specific condition or developing devices for a specific problem, which only covers some limited aspects of home healthcare. A comprehensive solution for in-home healthcare is still missing. A desirable system should be capable of taking care of the patients from all aspects, covering personalized medication, vital signs monitoring, on-site diagnosis and interaction with remote physicians. In addition, the existing systems seldom integrate new materials or apply new manufacturing approaches, which are always the key elements for bringing new devices or solutions into healthcare fields. By taking the aforementioned issues into consideration, an intelligent home-based healthcare IoT system, iHome Health-IoT, is proposed in this paper. Fig. 1 illustrates the concept of the iHome Health-IoT System. An intelligent medicine box (iMedBox) serves as a home healthcare gateway. IoT devices (e.g., wearable sensors, intelligent medicine packaging (iMedPack), etc.) are seamlessly connected to the iMedBox via a heterogeneous network which is compatible with multiple existing wireless standards. The body-worn Bio-Patch can detect and transmit the user's bio-signals to the iMedBox in real time. The iMedPack is connected with the iMedBox via a RFID link to assist the users with their prescribed medication. All the collected information is interpreted, stored and 


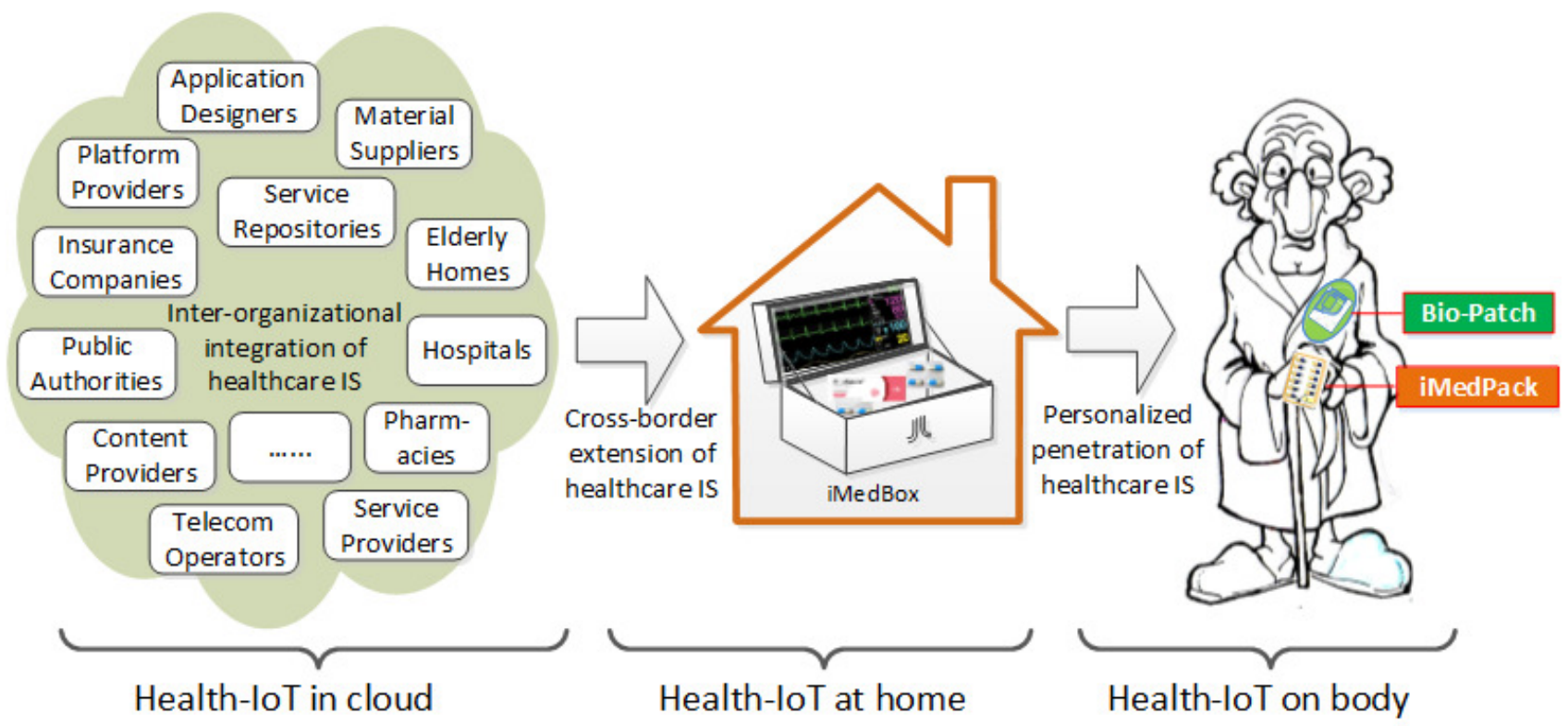

Fig 2. The Integrated Healthcare Information Systems enabled by the proposed iHome Health-IoT system.

displayed locally on the iMedBox. The processed information can also be forwarded to the Health-IoT network for a clinical diagnosis or further analysis.

One major contribution of the proposed iHome Health-IoT system is that it dramatically expands the scope and coverage of traditional Healthcare Information Systems (HIS), extending from a confined hospital environment to a patient's home and body, thus makes it possible to fully realize Integrated Healthcare Information Systems (IHIS) as introduced in [30] and [31]. By doing so, the overall healthcare system could be optimized at the top level, turning from the conventional Enterprise Resource Planning into the Entire Resource Planning [30]. As shown in Fig. 2, this paradigm shift includes three aspects:

- Inter-organizational integration of HIS

The backbone of the iHome Health-IoT system integrates the information systems (ISs) [32] of all the stakeholders that are involved in the value chain of Health-IoT. The emerging cloud computing technology [33] has offered a feasible environment for such inter-organizational integration towards the so-called Health-IoT-in-Cloud.

- Cross-border extension of HIS

The in-home terminal of the iHome Health-IoT system, iMedBox, acts as a bridge with the in-home healthcare devices. By deploying specific applications in the iMedBox, the ISs of the stakeholders can be seamlessly extended to a patient's home. Thus, the IHIS both comes within the context of enterprises and within the context of consumers' homes as the so-called Health-IoT-at-Home. This is consistent with the aforementioned trend of healthcare services which are transforming from hospital-centric to home-centric [2].

- Personalized penetration of HIS

Personalized services will be the ultimate form of future healthcare. The ultra-low-power and low-cost wearable biomedical devices, e.g., Bio-Patch, enable the personalized access of HIS to patients' bodies towards a so-called Health-IoT-on-Body. Therefore, the information and communication of HISs can be managed at the level of the body of a specific individual.

Considering the present and future importance of IHIS and IoT in e-health field, we developed the Health-IoT platform which can well find its applications in patients' home and nursing home scenarios. The proposed system takes the advantages of System-on-Chip (SoC) technology, material technology, and advanced printing technology, to build a patient-centric, self-assisted, fully-automatic intelligent in-home healthcare solution. The functions developed can be applied in various health-related scenarios, including environmental monitoring, vital signs acquisition, medication management, and healthcare services. This article describes the development of several smart devices, including Bio-Patch, iMedPack, and iMedBox to realize the vision of iHome Health-IoT.

\section{IHOME HEALTH-IOT SYSTEM}

\section{A. Network Architecture of iHome Health-IoT System}

The network architecture of iHome is shown in Fig. 3. It consists of three network layers: smart medical service layer, medical resource management layer, and sensor data collecting layer.

A smart medical service layer is directly linked to professional medical facilities such as hospitals, emergency centers, and medicine supply chain. For example, doctors can efficiently manage a large group of patients. They can inspect the medication history as well as the physiological status history of a specific patient, make further analysis of a suspicious portion of patient's bio-signals (e.g., ECG) and based on that 


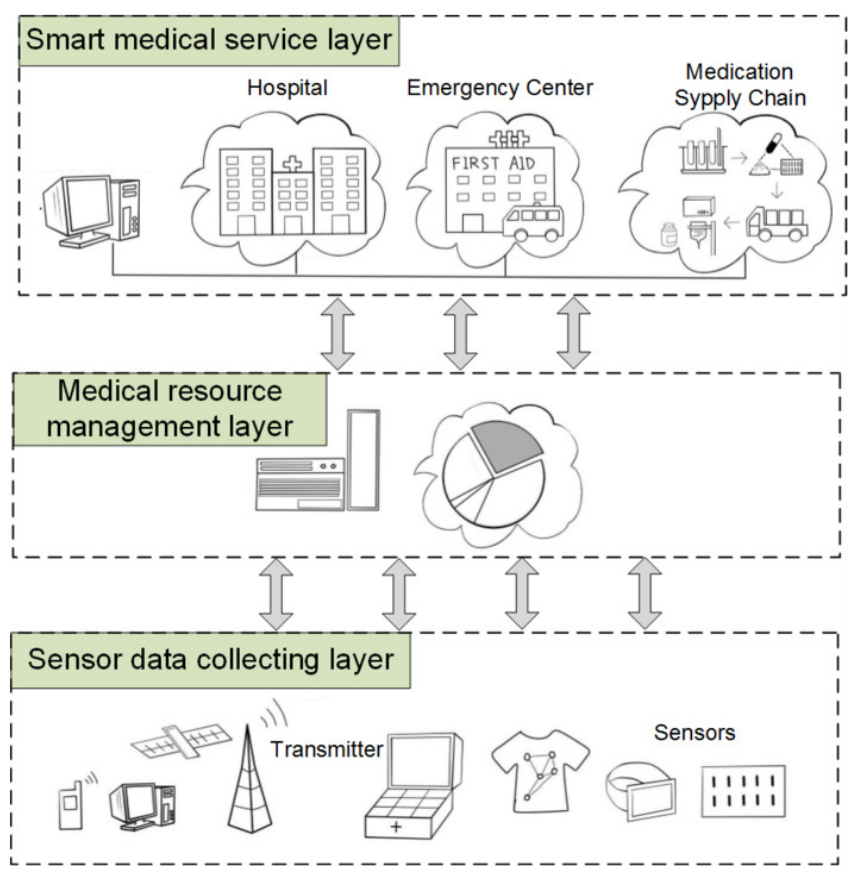

Fig. 3. Three-layer structure of the proposed iHome Health-IoT system.

make a new e-prescription accordingly. Also, the doctors can perform an overall examination of a patient group by using dedicated software which automatically analyzes the variation of an individual patient's physical condition over a period of time, for example, one week or one month. Subsequently, the doctors can easily identify the patient group whose health conditions have improved, and make them aware of their progress. Both patients and their family may feel reassured which helps build positive loops into rehabilitation and self-care.

The medical resource management layer works as a transition auxiliary layer, which involves the administration and management of medical resources in an efficient manner and facilitates the smooth operation of the iHome system. In this layer, cloud computing and services are available to health and life science providers, providing an efficient way for data security and patient privacy protection.

The sensor data collecting layer is the basis of the entire network. It consists of data sensing and acquisition devices, local computing and processing units, data storage devices, and wired/wireless transmitting modules. It is a multi-standard wireless sensor platform, compatible with different wired/wireless protocols, such as Ethernet, RFID, Zigbee, Wi-Fi, Bluetooth, and 3G/4G network. With this three-layer iHome Health-IoT system, interaction between clinical professionals and home-stay patients can easily take place on demand or on a regular basis.

\section{B. Architecture of the iMedBox}

The iMedBox is the central platform of the iHome Health-IoT system. The inspiration for the iMedBox comes from the traditional in-home medicine container. The difference

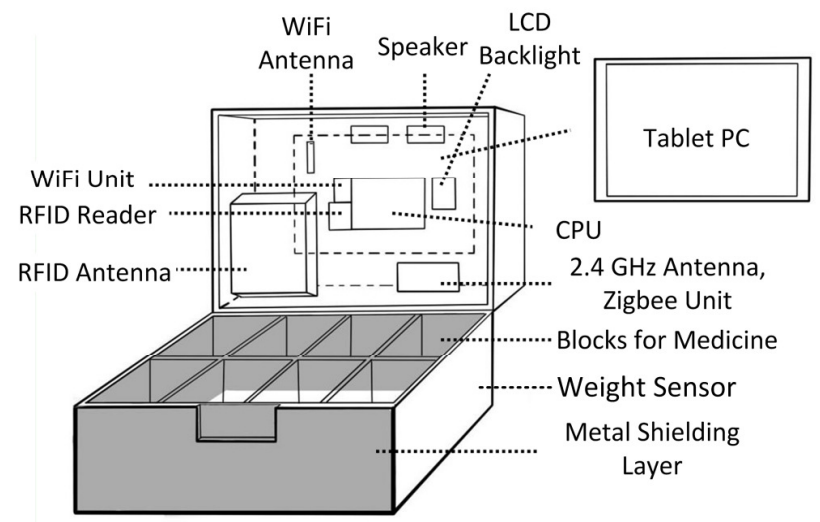

Fig. 4. Hardware architecture and interfaces of the iMedBox.

lies in the fact it is equipped with a high performance and open platform-based tablet PC and wireless transmission units, so the iMedBox is fully functional as a medication inspector, and an on-site examiner for daily monitoring [34]. The building blocks and interfaces of the iMedBox are shown in Fig. 4. An ultra-high frequency (UHF) RFID reader, a high frequency (HF) RFID reader, a Wi-Fi unit, a Zigbee receiver, and a tablet PC with extension ports are embedded into the lid. A high resolution weight bridge sensor is integrated in the bottom of the iMedBox to track the weight variation of the medicine stored in the box, and based on which the dose of medication taken by the patient can be calculated.

Wearable medical sensors (e.g., Bio-Patch), intelligent medicine packages, as well as the sensors/devices from third parties can be connected to the iMedBox via various wireless technologies. The iMedBox can serve as an in-home healthcare gateway to gather patients' physiological information, and it can deliver a variety of services such as on-site analysis, health social network, telemedicine, emergency and medication management services.

\section{InTELligent Medicine PACKAGING}

Nowadays, for senior citizens and patients with chronic diseases, it is critical to follow the doctors' advice to take their prescribth [35]. An intelligent medication administration system is desirable to timely remind and dispense the medicine to individuals, and in the meantime, register and track their medication history.

\section{A. Intelligent Medicine Packaging using RFID and CDM}

In order to solve the medicine misuse problem, improve pharmaceutical non-compliance situation, and make the daily task as easy and smart as possible, an intelligent medicine packaging (iMedPack) is proposed utilizing two key technologies: RFID technology and Controlled Delamination Materials (CDM).

In recent years, RFID technology has become more and more popular in the applications of ed medicine at the proper time. However, non-compliance with medication is becoming more prevalent [4]. The levels of non-compliance may be affected by psychological factors such as the patients' levels of anxiety, 


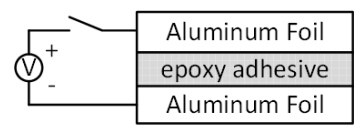

a) Unopen/Bond b) Open/Release

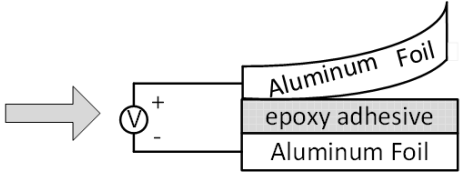

Fig. 5. The structure and principle of CDM, a) CDM in sealed status, b) Aluminum foil can be easily peeled off after applying a 10-50V DC voltage for a short period.

motivation to recover, attitudes towards their illness, as well as the fact that many senior citizens suffering from amnesia often forget to take the prescribed medicine on time. Prescribing clinicians frequently do not often detect or ask about non-compliance and are not always good at recognizing when patients stop taking their medication. If possible, it is important to maintain routine contact with the doctor to discuss, among other things, compliance issues. However, this is not as easy as it sounds. Moreover, the misuse and abuse of prescription medication can cause a range of adverse drug reactions, sometimes even leading to deamanufacturing industries, logistics providers, supply chain management, retail outlets, banks, location tracking and process detection [36]. With the rise of Gen 2 protocol, RFID tags are becoming more powerful in terms of larger memory, faster reading speed, higher information security [37]. Therefore, RFID tags provide more opportunities for commercial applications.

$\mathrm{CDM}$ is a thin layer of epoxy adhesive sandwiched between two pieces of metal substrate like aluminum foil [36], as shown in Fig. 5. In a bonded state, as shown in Fig. 5a, the strong adhesion of the glue epoxy can have a tension as large as 211 $\mathrm{kg} / \mathrm{cm}^{2}$ in ambient environment. The adhesion disappears quickly after a 10-50 V DC voltage is applied, and subsequently, the interface is released, as shown in Fig. 5b. In previous work, we applied a series of measurements to quantitatively study the electrical characteristics of CDM which can be summarized as follows [36].

1) The opening time will decrease monotonically along with an increase in DC voltage from $5 \mathrm{~V}$ to $30 \mathrm{~V}$.

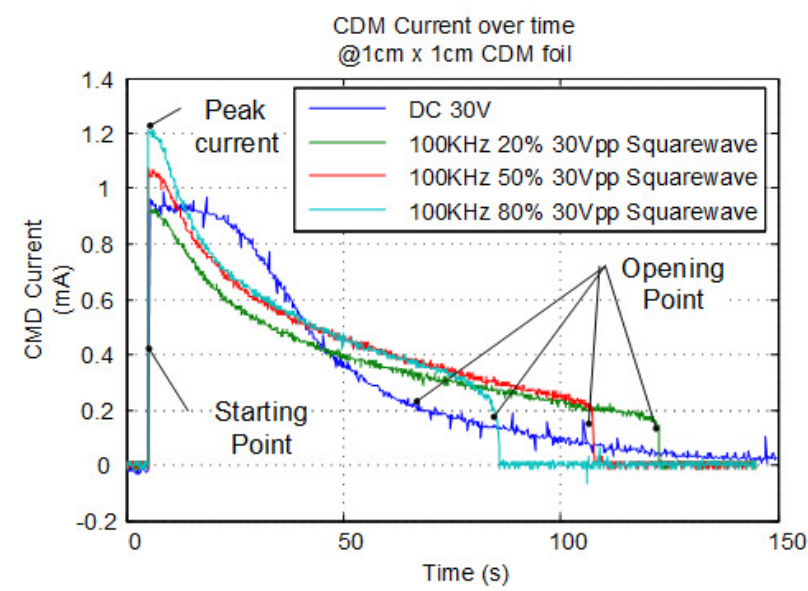

Fig. 6. Current waveform of $1 \mathrm{~cm}^{2} \mathrm{CDM}$ driven by $100 \mathrm{kHz}$ square-wave with $\mathrm{Vpp}$ of $30 \mathrm{~V}$ and duty cycle of DC, $80 \%, 50 \%$ and $20 \%$.

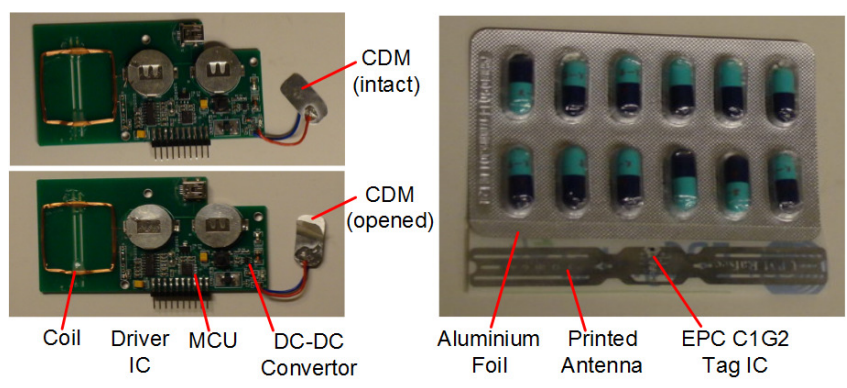

Fig. 7. Examples of an iMedPack prototype.

2) The opening time is independent on the material size.

3 ) The delamination current waveform of $1 \mathrm{~cm}^{2} \mathrm{CDM}$ is shown in Fig. 6. With a DC power supply of $30 \mathrm{~V}$, the delamination time of CDM is tens of seconds, and the current is in the range of hundreds $\mu \mathrm{A}$, within the safe range.

4) With a $100 \mathrm{kHz}$ square-waves power supply (Vpp of $30 \mathrm{~V}$ ), the opening time monotonically varies with different duty cycles as shown in Fig. 6.

Due to the low-power consumption, quick response, and electrically-controlled delamination features, we combined $\mathrm{CDM}$ into an aluminum foil covered capsule package. We thereby substituted the original single-layer adhesive aluminum layer with a sandwich-structured CDM to seal the packages. Combined with advanced RFID technology, an iMedPack was implemented. An RFID tag is attached along the edge of the medicine package, and connected to the CDM opener, as shown in Fig. 7 [38]. The RFID tag is wireless-powered by the reader embedded in the iMedBox. The tag can convert the near-field magnetic wave emitted by the reader into a DC supply, and an integrated charge-pump circuit can boost the DC voltage to around $30 \mathrm{~V}$ for CDM opening. The RFID always keeps the charge-pump module shut down until it receives an opening-command issued by the iMedBox.

During this process, the iMedBox works as a home healthcare platform. According to the clinical prescriptions stored inside, it timely sends commands to the targeted iMedPack, and in the meantime, it emits magnetic waves to power the passive iMedPack. In return, the selected iMedPack sends back local information, e.g., packaging's ID, the number of medicine slots opened, and the number of intact slots, and acts according to the commands received, e.g., to open specific medicine slots. With the combination of the iMedBox with the iMedPack, the system can give timely reminders to patients about their medication, and precisely control the type and amount of prescribed drugs, thus avoiding medication misuse and abuse.

\section{UNOBTRUSIVE BIO-SENSOR}

Biological signals, such as ECG and electroencephalograms (EEG), are the most commonly used vital tools for monitoring patients' physical condition and diagnosing diseases [39]. In particular for premature heart attacks, a very high proportion of lethal attacks happen during sleep or daily activities. The sooner the symptom is detected, the earlier medical treatment and the 


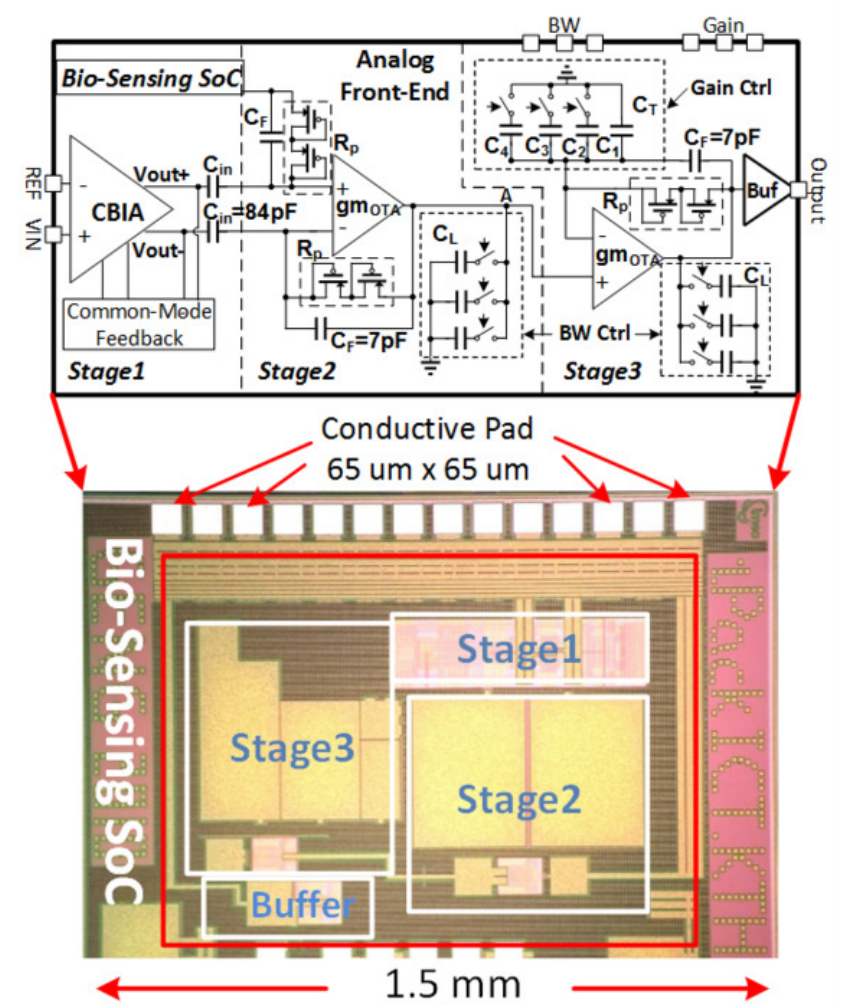

Fig.8. Schematic and microphotograph of the three-stage bioelectrical amplifier.

better prognoses can be made for the patients. However, besides heart diseases, many chronic diseases are asymptomatic or intermittent which lead to difficulties in accurate detection during a short visit to a hospital. Therefore, long-term continuous health monitoring is essential for detecting and treating diseases.

Existing continuous monitoring systems (e.g., Holter system, etc.) are usually uncomfortable and inconvenient for long-term use, due to their physical limitations, e.g., large size, rigid package and twisted wires, etc [40]. In previous work, a wearable ECG sensor node was developed using off-the-shelf components, which successfully detected ECG signals [41]. However, this sensor node was strap-based. In order to ensure good physical contact between embedded electrodes and human body, the strap had to be tightly pressed against the user's chest, which made it uncomfortable for long-term use. A new generation of healthcare devices should feature: 1) comfortable and unobtrusive properties, 2) low power consumption therefore long battery life, and 3) an affordable price.

\section{A. Concept of the Flexible Bio-Patch}

A desirable wearable bio-sensing device for continuous measurement should be ideally ultra-thin, small and lightweight. In order to fulfill all these requirements, the Bio-Patch is proposed as an essential data-recording device within the Health-IoT system as shown in Fig. 1. The Bio-Patch monitors a variety of physiological parameters simply by placing it on the relevant parts of the body. It converts bio-signals into a digital format and wirelessly transmits the data to the iMedBox platform for storage or real-time processing/display. Compared with existing health monitoring devices, two key technologies have been employed in the implementation of the Bio-Patch: 1) fully-integrated high-performance $\mathrm{SoC}$, and 2) large-scale cost-effective inkjet printing technology. Flexible and thin substrates have been adopted, such as paper and plastic, to improve user comfort.

A customized Bio-Sensing SoC is designed to sense, amplify, digitize, and process the bio-signals. Compared with devices or systems made from off-the-shelf components, the customized silicon chip compacts power-efficient circuit modules, such as amplifier, analog-to-digital convertor, digital controller, and wireless transmission modules, on to the same chip. A full integration of optimized circuit blocks enables the miniaturization of the physical size of the device as well as reducing power consumption [42]. These features are the key elements to realize the tiny but long-lasting healthcare device. A three-stage low-power, low-noise bioelectrical amplifier has been developed. The schematic and architecture of the amplifier are illustrated at the top of Fig. 8. A microphotograph of the fabricated Bio-Sensing SoC is shown at the bottom of Fig. 8 . The chip is designed to have programmable bandwidth $(80 \mathrm{~Hz}$ $1.5 \mathrm{kHz})$ and gain $(45 \mathrm{~dB}-63 \mathrm{~dB})$ by tuning the on-chip integrated load capacitors via external switches. Therefore it can be configured to sense a variety of bio-electric signals, including ECG and EEG. In addition, a temperature sensor is integrated on-chip, which can monitor a patient's body temperature, a commonly-measured parameter indicating people's health status. The implemented three-stage bioelectrical amplifier totally consumes $2.3 \mu \mathrm{A}$ current from a $1.2 \mathrm{~V}$ supply. A detailed report regarding the circuit design as well as measurement results can be found in [42]. In order to make the Bio-Patch small and soft enough to be stuck on the body, hybrid integration technology is adopted. The Bio-Patch is implemented by mounting and interconnecting the bare Bio-Sensing SoC and other electronics on a flexible foil that improves comfort when the device is wore on the body.

\section{B. Inkjet Printed Electrodes and Interconnections}

Flexible electronics are considered a promising solution for next-generation consumer electronics because of their unique features. They are thin, lightweight, flexible, and compatible with cost-effective substrates and manufacturing techniques [44], [45]. They enable many attractive applications, such as flexible displays, e-textiles, RFID tags, and bio-sensors [46]. Printing technology is considered to be a revolutionary approach for cost-effective manufacturing such flexible electronic devices [47]. Inkjet printing technology uses cartridges to inkjet small amounts of functional inks directly and accurately on to a substrate [48]. With the development of nanotechnology, new materials, such as inorganic nanoparticles, carbon nanotubes, and graphene inks, have been developed to build high performance components and large-area electronics that are thin, light weight, flexible or stretchable [49]. Due to the features of additive and digital manufacture processes, inkjet printing is mask-free and free of material waste, therefore it is a 

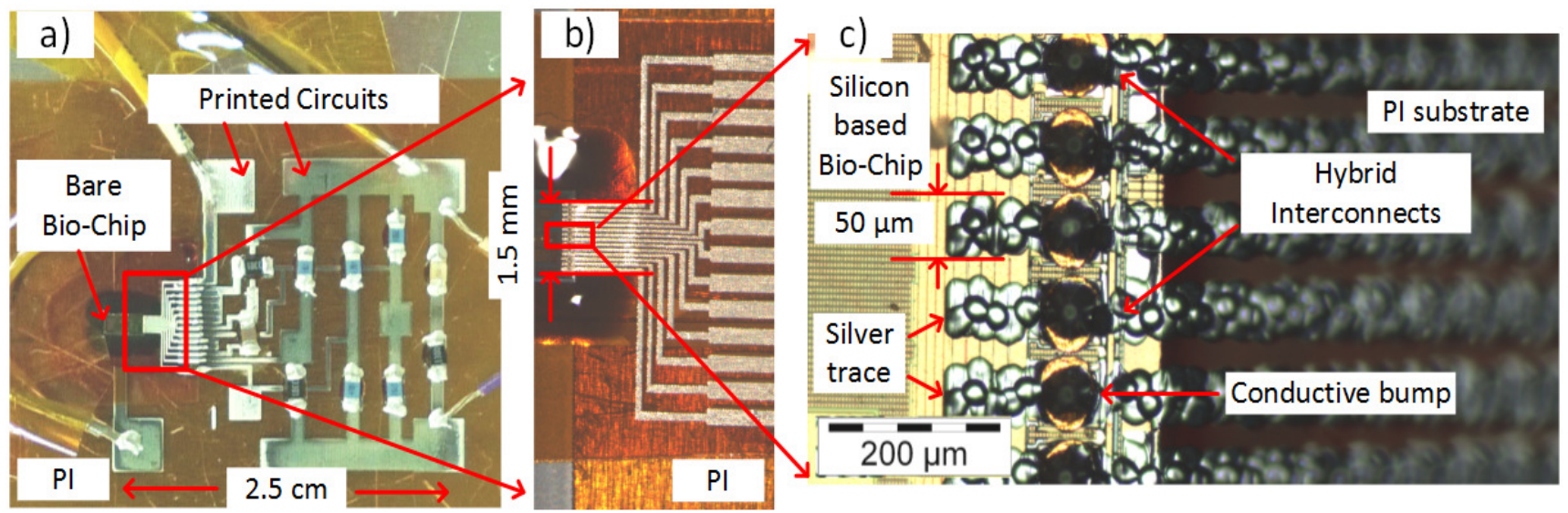

Fig.10. Hybrid integration of Bio-Patch using inkjet printing technology.

'green' and cost-effective assembly approach for electronic systems [50]. In addition, it is roll-to-roll compatible, enabling mass production with the possibility of further lowering the production cost. The print head does not need to contact the substrate, so it is capable of printing on multiple substrates, such as paper [51] and plastic [52]. This suggests a free selection of ultra-thin and flexible substrates according to the application requirements and makes the final device thin, light and flexible.

In this work, inkjet printing technology is used in the manufacturing of tiny-size healthcare monitoring devices. The performance and feasibility of inkjet-printed electrodes and circuit boards have been reported in [53]. Some examples are shown in Fig. 9, including printed flexible electrodes and interposer lead-frames for bendable circuit packaging. Through a series of ECG in-vivo tests, the electrical performance and design rules have been studied and summarized below [53], which help to build an electrodes pair with optimized size and distance:

1) The signal quality of ECG captured by printed electrodes is comparable with commercial pre-gelled electrodes of the same size and distance;

2) The amplitude of detected ECG signal decreases along with the shrinking of electrode size (diameter from $15 \mathrm{~mm}$ to 3 $\mathrm{mm}$ ) and the distance between electrodes (from $10 \mathrm{~cm}$ to $2 \mathrm{~cm}$ ).

In previous work [50], a successful integration of a packaged chip has been reported using inkjet printing technology. However, in order to further miniaturize the physical size of Bio-Patch, instead of using a standard-packaged SoC chip, investigations are made to achieve the direct integration of a
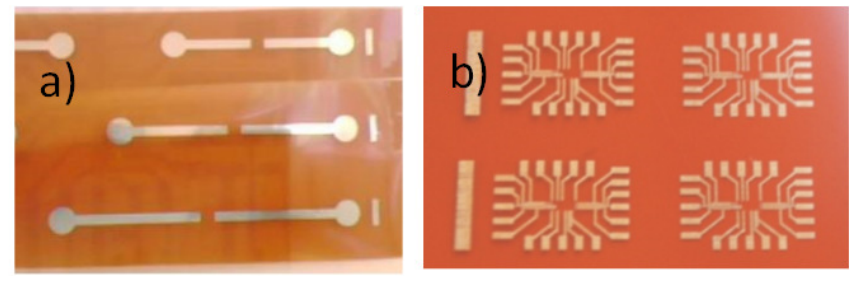

Fig. 9. Examples of inkjet printed pattern on Polyimide substrate, a) Inkjet printed electrodes and b) bendable interposers for chip packaging. bare silicon die on to flexible substrates, named Silicon-on-Flex. This presents great challenges to the integration approach due to the following two aspects: 1) the pad size on the silicon die is as small as $65 \mathrm{um} \times 65$ um with a pitch of 90 um. During the integration process, adjacent pads can easily get short-circuited; 2) the flexible substrate is soft, and moreover cannot stand very high temperatures. So traditional integration methods, such as wire bonding and solder balls, are not suitable in this case. Owing to the features of high accuracy and fine resolution, inkjet printing technology can not only be used to manufacture electrodes and circuit boards, but also be used as an integration approach. Therefore, the whole pattern could be printed at once, including the electrodes, the circuit board, and the interconnections connecting the pads on the die with the circuits on the flexible foil. It is worth mentioning that, by merging the steps of printing the circuit and electrodes into a single step of system integration, the whole manufacturing process for a device is further simplified.

Fig. 10 shows one Bio-Patch prototype based on the hybrid Silicon-on-Flex integration. The width of inkjet-printed interconnects can be as thin as $50 \mathrm{um}$. In total 14 parallel interconnects have been successfully printed within a narrow space of $1.5 \mathrm{~mm}$ (one edge of a bare die) without any open-circuit or short-circuit.

\section{Implementation and Experimental Results of Bio-Patch}

By integrating the customized bio-sensing SoC, the optimized electrodes, and the other circuits, the miniaturized Bio-Patch prototype was implemented for single-channel ECG measurement, as shown in Fig. 11a. Including the electrodes and the coin battery assembled on the back, the total size of the Bio-Patch is $4.5 \mathrm{~cm} \times 2.5 \mathrm{~cm}$ with a thickness of $2 \mathrm{~mm}$. The thickness could be further reduced to $1 \mathrm{~mm}$ if a soft battery (e.g., an Enfucell printed battery) is used. The hybrid integration approach of Silicon-on-Flex makes the developed Bio-Patch small, ultra-thin, and bendable. With the help of bio-compatible adhesive tape, the implemented Bio-Patch can be easily attached on to the patient's chest. Due to the use of thin flexible polyimide substrate, it works like a typical 'Band Aid'. The ECG signal captured by the Bio-Patch is shown in Fig. 11b. 


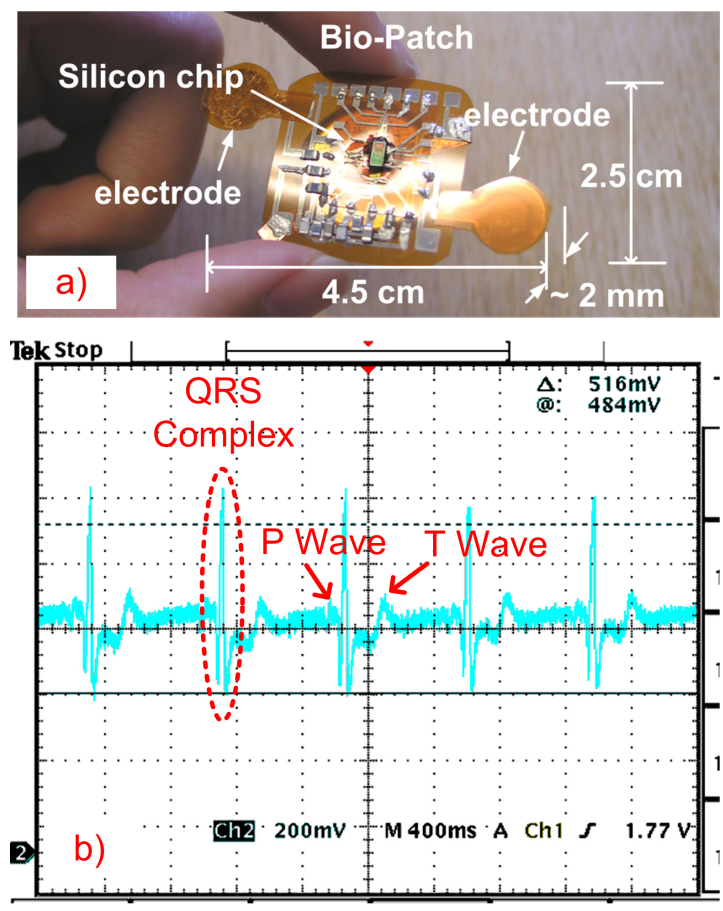

Fig. 11. Miniaturized Bio-Patch for single-channel ECG measurement.

Influenced by $50 \mathrm{~Hz}$ power-line interference, a slight noise can be observed from the measured ECG signal, but the key features of ECG are clearly visible.

\section{SYSTEM INTEGRATION AND PROTOTYPE DEMONSTRATION}

The iMedBox platform is built based on the integration of the iMedPack and smart sensors via various wireless links. The focus is to regulate and optimize the accessibility of medical drugs and efficiently provide home-based healthcare services. The developed iHome IoT system connects the individual home environment with hospital, emergency center and other medical facilities and provides remote prescription and medication non-compliance service. The key innovation and functionality include:

\section{A. Remote Prescriptions}

An iMedCard is an RFID tag based medical card with a unique ID number linked to each user. The user registration process is shown in Fig. 12a. When a user with an iMedCard comes within the reading range of the iMedBox, the iMedBox will identify the user by comparing the ID number with the pre-stored key. If these two numbers do not match, the iMedBox will turn off, which means the iMedBox does not contain the medicine prescribed for this user. If two ID numbers match, the iMedBox will be activated and the relevant prescription will be automatically downloaded from the database through a wired or wireless link. When the user is registered into the iMedBox, the prescription page in Fig. 12b will show up on the graphic user interface (GUI), containing user information as well as the detailed prescription. From the remote physician's perspective, it is very convenient to make or update a prescription for a
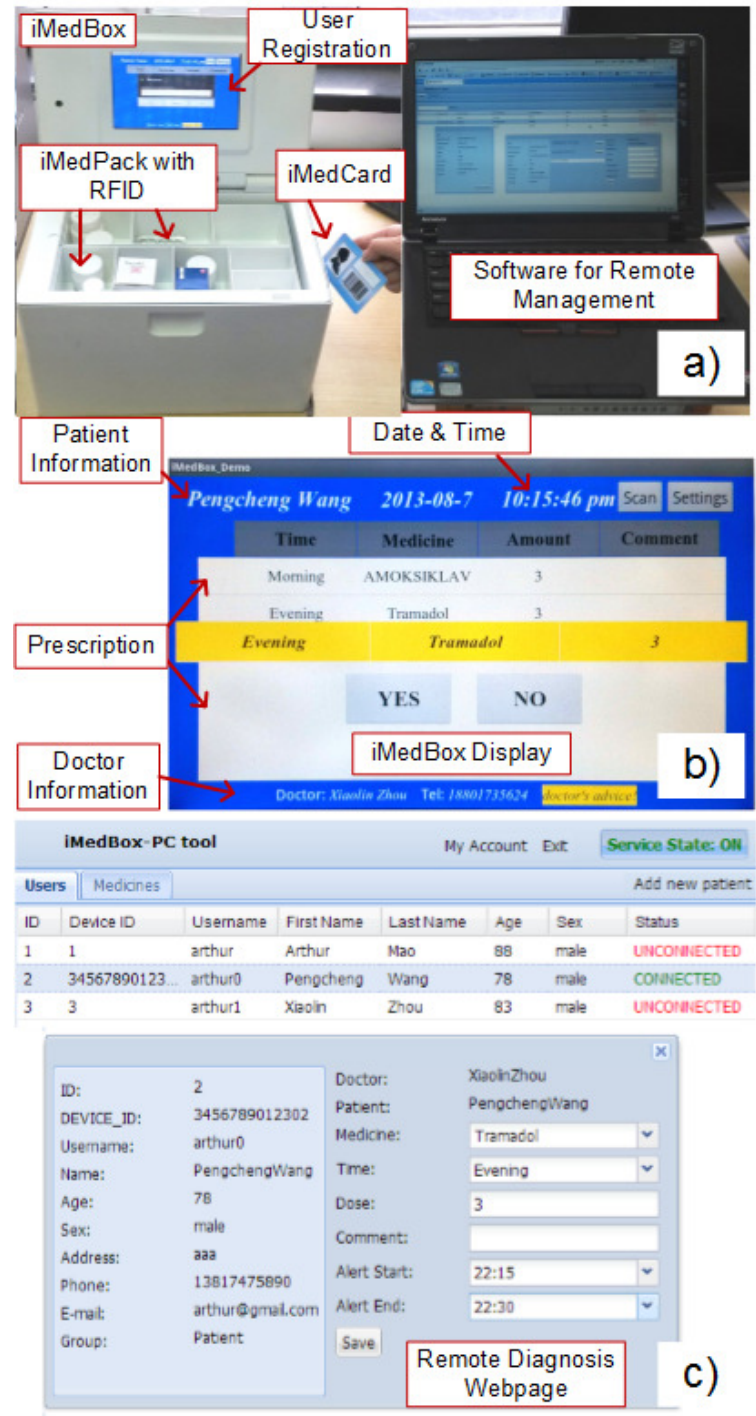

Fig. 12. Remote Prescription and medication management of iHome Healthcare System.

selected user. The doctor only needs to upload the prescription along with his/her ID into the database through the Internet using the website as shown in Fig. 12c. When the database is updated, the iMedBox will synchronize automatically.

\section{B. Medication Reminder}

The iMedPack is sealed by CDM and integrated with an RFID tag as introduced in the previous section. The medicines are kept in the iMedBox just as in a normal in-home medication unit. The iMedBox recognizes and registers the medicine information by the RFID number, and meanwhile, compares the medicine with the prescription. According to the stored prescription, the controlling logics in the iMedBox will manage when and how many individual packages should be opened. When the time comes, the iMedBox will activate an audio or video reminder (taking into consideration the difficulty of reading prescription labels for patients with a visual impairment or hearing loss), including which kind of medicine to take, and 
how much to take. At the same time, the selected iMedPack will turn on the charge pump to provide the CDM with $30 \mathrm{~V}$ for opening it.

\section{Medication Non-compliance Control}

According to the electrical characteristics of CDM shown in Fig. 6, the bonding strength of the CDM glue decreases along with the duration of electrical stimulation, while at the same time, the pass-through current also decreases. When the current drops below $0.2 \mathrm{~mA}$, the bonding strength is relatively weak, so a slight touch can peel it off. Therefore the ready-point is defined as the time when the pass-through current drops below $0.2 \mathrm{~mA}$. The current keeps on decreasing infinitely close to zero. It will never reach zero until the user peels off the package and take the pills. Based on this electrical characteristic, the iMedPack can precisely identify the time when the specific medicine is taken and then forward this information back to iMedBox via the RFID link.

An alternative method is available by tracking or measuring the remaining doses of medicine left in the bottles. In this case, an RFID tag is attached underneath each bottle. When the bottle is taken from the box, the short-range RFID reader will recognize the event that a bottle has been taken from the iMedBox and record its ID number which is directly linked to the type of medicine picked up. After the user puts the bottle back, the iMedBox measures the weight difference of the bottle before and after the event, using the weight bridge sensor located at the bottom of the iMedBox. Based on the weight difference, the iMedBox can calculate how many pills have been taken.

\section{Intelligent Analysis and First Aid Alarm}

The elderly are more likely to forget to take or fail to properly take the medicine as prescribed. The following cases may be common: the user takes a wrong medicine, takes too much or too little of a specific medicine, takes the medicine at the wrong time, or adverse drug reactions/interactions happen with the possibility of causing death. According the severity in each case, various levels of alarms should be triggered. The iMedBox performs an intelligent analysis by automatically comparing the recorded medication time and amount with the doctor's prescription. If a mismatch happens, the iMedBox will trigger an alarm. As shown in Fig. 13a, the alarm will firstly show up on the iMedBox's screen. If the mistake is not corrected within 10 minutes, a text message will be sent to the doctor through the embedded 3G module. The message includes user information and a description of the non-compliance, as shown in Fig 13b. Subsequently, the doctor can log into the database via a remote terminal, and make an estimation to decide whether it is necessary to contact the user immediately or deliver the information to the emergency center. The medication history is regularly uploaded and saved in a remote server. The doctor can take it as a reference for the next prescription.

By connecting the wearable Bio-Patch with the iMedBox, the iHome Health-IoT system is capable of monitoring and analyzing the vital signs. The bio-signals sampled, such as ECG

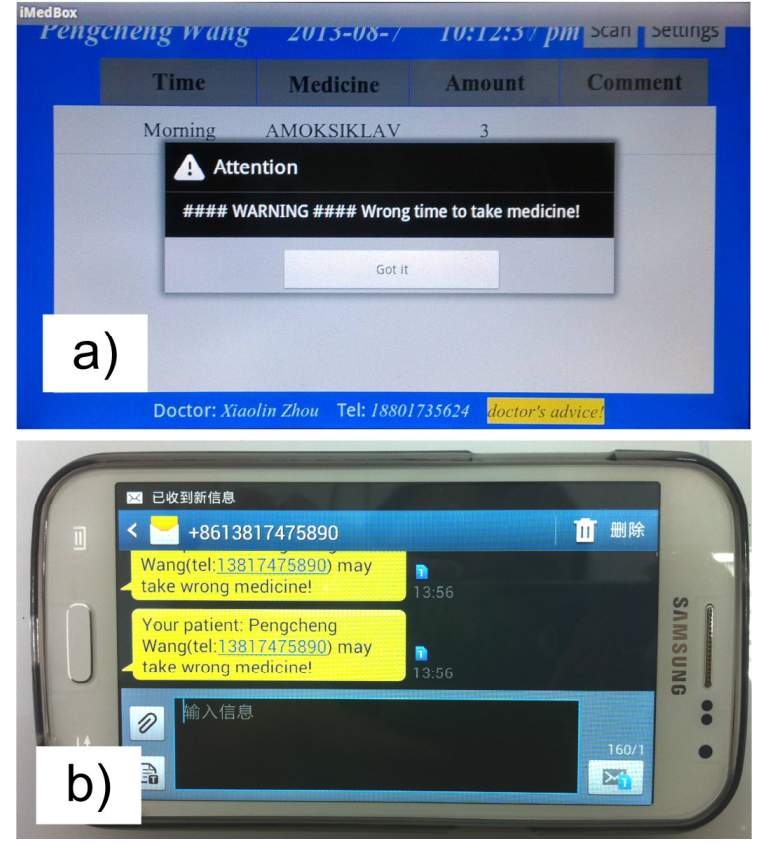

Fig. 13. The First Aid Alarm of the iHome Healthcare System: a) alarm shown on iMedBox screen, b) SMS sent to clinical professionals.

and body temperature, are digitized and wirelessly transmitted to the iMedBox, which stores the data, and performs real-time signal processing and display. Based on the recorded ECG signal from Bio-Patch, the user's heart rate information can be extracted, and a heart rate variability analysis can be performed on a regular basis. Once a continuous abnormal heart rate is detected, the iMedBox will trigger an alarm. If the patient's abnormal heart condition has not recovered within a certain time period, e.g., 10 minutes, the iMedBox will automatically send out a text message to the doctor describing the situation. The doctor will then decide whether or not to contact the patient's relatives or delivery the case to an emergency center. Audio and video communication between the patient and the doctor is also available through the iMedBox to support an on-site diagnosis.

\section{CONCLUSION AND FUTURE WORK}

In recent decades, the rapid growing of aging population has been a challenge to global healthcare systems [1]. Many countries have been active in undergoing hospital restructuring through optimizing medical resources and increasing the use of home healthcare [2], [54], [55]. IoT now has been recognized as a revolution in ICT and is expected to be applied to many industrial sectors including healthcare [56]-[71]. This paper presents an IoT-based intelligent home-centric healthcare platform (iHome system), which seamlessly connects smart sensors attached to human body for physiological monitoring and intelligent pharmaceutical packaging for daily medication management. It also offers multiple opportunities to adapt a wide variety of e-health applications with minimum changes. Examples include the scenario of assisted living for people with disabilities, where users can interact with smart objects, such as 
home appliances, and environmental sensors etc., deployed in a home environment to ensure their health and well-being.

The proposed iHome system consists of three key blocks, including the iMedBox, the iMedPack, and the Bio-Patch. It also involves different aspects of the healthcare information system, from the hospital, emergency center, to user's home, body and even medicine. The iMedBox serves as a home healthcare station providing strong interoperability and IoT network connectivity. By leveraging CDM material and RFID technology, iMedPack offers a promising solution for the medication non-compliance problem by automatically reminding the user and dispensing a certain amount of medicine on time according to the on-line prescription. In order to continuously and unobtrusively monitor a user's vital signs, a miniaturized flexible Bio-Patch has been developed. An ultra-low power, tiny-size, application-specific integrated circuit has been developed to measure ECG and body temperature. Inkjet printing technology offers a cost-effective solution for manufacturing electrodes and Silicon-on-Flex integration. A wearable Bio-Patch has been developed for bio-signal acquisition by leveraging the advantages of hybrid integration of silicon-based electronics and printed electronics.

By connecting the iMedPack, Bio-Patch, and the back-end services through a wireless link, the iMedBox can deliver various services, including real-time bio-signal monitoring, local analysis and alarm, remote diagnosis and prescription, as well as medication non-compliance control. The iHome Health-IoT system combines the health social network, telemedicine, emergency and medication management services. It will speed up the transformation from Hospital-Centric medical treatment to Home-Centric healthcare, and finally bring about ubiquitous and personalized healthcare.

The viability of the Health-IoT platform has been proved by conducting a series of in-field tests, involving bio-sensing, data retrieval, medication reminder and alarm, multi-standard communication capability test, etc. However, limitations still exist in the implemented system. For instance, the implemented Bio-Patch is bendable, but in some extreme cases, it may not survive a sharp-edge folding because the attached silicon chip may peel off. In addition, the current interactions between a user and the remote physician are mainly based on short messages, phone calls or simple notices on the iMedBox's GUI. A user-friendly and interactive multimedia interface is desirable for a better user experience. Future work will comprise further enhancing Bio-Patch's mechanical and electrical reliability by laminating a thin plastic insulation layer over the patch to protect the conductive traces. Enriching the iMebBox's GUI with more user-friendly functions and exploring new application scenarios for this Health-IoT platform are also open issues to work on.

\section{ACKNOWLEDGMENT}

The authors would like to thank Xixu Wang and Junjie Mao from Fudan University, Shanghai, China, for their contributions on the software design of the iMedBox.

\section{REFERENCES}

[1] Department of Economic and Social Affairs of the United Nations. 2009. World Population Ageing 2009, New York: United Nations.

[2] Z. Pang, "Technologies and Architectures of the Internet-of-Things (IoT) for Health and Well-being," PhD Thesis, Royal Institute of Technology (KTH), Stockholm, Sweden, 2013.

[3] C. E. Koop, et al., "Future delivery of health care: Cybercare," IEEE Engineering in Medicine and Biology Magazine, vol.27, no.6, pp.29-38, Nov. 2008.

[4] B. Schuz, et al., "Medication beliefs predict medication adherence in older adults with multiple illnesses," Journal of Psychosomatic Research, vol. 70, no. 2, pp. 179-187. 2011.

[5] K. Ashton "That 'Internet of Things' Thing," RFID Journal, Jun. 2009.

[6] S. Li, L. Xu, and X. Wang, "Compressed Sensing Signal and Data Acquisition in Wireless Sensor Networks and Internet of Things, "IEEE Transactions on Industrial Informatics vol.9, no.4, pp. 2177-2186, Nov. 2013.

[7] E. Welbourne, et al., "Building the Internet of Things Using RFID: The RFID Ecosystem Experience," IEEE Internet Computing vol.13, no.3, pp.48-55, Jun. 2009.

[8] G. Kortuem, F. Kawsar, D. Fitton, and V. Sundramoorthy, "Smart objects as building blocks for the Internet of things," IEEE Internet Computing, vol.14, no. 1 , pp.44-51, Feb. 2010 .

[9] S. Tozlu, M. Senel, W. Mao, and A. Keshavarzian, "Wi-Fi enabled sensors for internet of things: A practical approach," IEEE Communications Magazine, vol.50, no.6, pp.134-143, Jun. 2012.

[10] A. J. Jara, M. A. Zamora-Izquierdo, and A. F. Skarmeta, "Interconnection Framework for mHealth and Remote Monitoring Based on the Internet of Things," IEEE Journal on Selected Areas in Communications, vol. 31, no.9, pp.47-65, Sep. 2013.

[11] S.-Y. Lee, L.-H. Wang, and Q. Fang, "A Low-Power RFID Integrated Circuits for Intelligent Healthcare Systems," IEEE Transactions on Information Technology in Biomedicine, vol.14, no.6, pp.1387-1396, Nov. 2010.

[12] X. Li, R. Lu, X. Liang, X. Shen, J. Chen, and X. Lin, "Smart community: an internet of things application," IEEE Communications Magazine, vol.49, no.11, pp.68-75, Nov. 2011.

[13] G. Broll, E. Rukzio, M. Paolucci, M. Wagner, A. Schmidt, and H. Hussmann, "Perci: Pervasive Service Interaction with the Internet of Things," IEEE Internet Computing, vol.13, no.6, pp.74-81, Nov. 2009.

[14] European Commission Information Society. 2008. "Internet of Things in 2020: A Roadmap for the Future." http://www.iot-visitthefuture.eu.

[15] A. Hande, and E. Cem, "Wireless sensor networks for healthcare: A survey," Computer Networks, vol. 54, no. 15, pp. 2688-2710, Oct. 2010.

[16] National Information Council. 2008. "Global Trends 2025: A Transformed World. US Government Printing Office." http://www.acus.org/publication/global-trends-2025-transformed-world.

[17] S. Li, L. Xu, and X. Wang, "A Continuous Biomedical Signal Acquisition System Based on Compressed Sensing in Body Sensor Networks," IEEE Transactions on Industrial Informatics, vol.9, no.3, pp.1764-1771, Aug. 2013.

[18] P. Castillejo, J.-F. Martinez, J. Rodriguez-Molina, and A. Cuerva, "Integration of wearable devices in a wireless sensor network for an E-health application," IEEE Wireless Communications, vol.20, no.4, pp.38-49, Aug. 2013.

[19] X.-F. Teng, Y.-T. Zhang, C. Poon, and P. Bonato, "Wearable Medical Systems for p-Health," IEEE Reviews in Biomedical Engineering, vol.1, no., pp.62-74, 2008.

[20] J. Morak, H. Kumpusch, D. Hayn, R. Modre-Osprian, and G. Schreier, "Design and Evaluation of a Telemonitoring Concept Based on NFC-Enabled Mobile Phones and Sensor Devices," IEEE Transactions on Information Technology in Biomedicine, vol. 16, no. 1, pp. 17-23, Jan. 2012.

[21] H. Baek, G. Chung, K. Kim, and K. Park, "A Smart Health Monitoring Chair for Nonintrusive Measurement of Biological Signals," IEEE Transactions on Information Technology in Biomedicine, Vol. 16, no. 1, pp. 150-158. Jan. 2011.

[22] R. Paradiso, G. Loriga, and N. Taccini, "A wearable health care system based on knitted integrated sensors," IEEE Transactions on Information Technology in Biomedicine, vol. 9, no. 3, pp. 337-344, Sep. 2005.

[23] D. Curone, E. L. Secco, A. Tognetti, G. Loriga, G. Dudnik, M. Risatti, R. Whyte, A. Bonfiglio, and G. Magenes, "Smart Garments for Emergency 
Operators: The ProeTEX Project," IEEE Transactions on Information Technology in Biomedicine, vol.14, no.3, pp.694-701, May 2010.

[24] S. Coyle, et al., "BIOTEX-Biosensing Textiles for Personalised Healthcare Management," IEEE Transactions on Information Technology in Biomedicine, vol.14, no.2, pp.364-370, 2010.

[25] C. R. Merritt, H. T. Nagle, and E. Grant, "Fabric-based active electrode design and fabrication for health monitoring clothing," IEEE Transactions on Information Technology in Biomedicine. vol.13, no.2, pp.274-280, 2009.

[26] M. Kaltenbrunner, et al., "An ultra-lightweight design for imperceptible plastic electronics," Nature 499, pp. 458-463. Jul. 2013.

[27] H. Fuketa, et al., "1 $1 \mu \mathrm{m}$-thickness 64-channel surface electromyogram measurement sheet with $2 \mathrm{~V}$ organic transistors for prosthetic hand control," IEEE International Solid-State Circuits Conference (ISSCC), pp.104,105, Feb. 2013.

[28] T. Someya, "Building bionic skin," IEEE Spectrum, vol.50, no.9, pp.50-56, Sep. 2013.

[29] H. Liu, "Exploring Human Hand Capabilities Into Embedded Multifingered Object Manipulation," IEEE Transactions on Industrial Informatics, vol.7, no.3, pp.389-398, Aug. 2011.

[30] L. Xu, "Enterprise Systems: State-of-the-Art and Future Trends," IEEE Transactions on Industrial Informatics, vol.7, no.4, pp.630-640, Nov. 2011.

[31] E. Xu, M. Wermus, and D. B. Bauman, "Development of an integrated medical supply information system," Enterprise Information Systems, vol.5, no.3, pp. 385-399, May. 2011.

[32] L. Li, L. Xu, A. Jeng, D. Naik, T. Allen, and M. Frontini, "Creation of environmental health information system for public health service: A pilot study," Information System Frontiers, vol. 10, no. 5, pp. 531-543, 2008.

[33] S. Hodges, S. Taylor, N. Villar, J. Scott, D. Bial, and P.T. Fischer, "Prototyping Connected Devices for the Internet of Things," Computer vol.46, no.2, pp.26-34, Feb. 2013.

[34] Z. Pang, Q. Chen, J. Tian, E. Dubrova, and L. Zheng, "Ecosystem analysis in the design of open platform-based in-home healthcare terminals towards the internet-of-things," 15th International Conference on Advanced Communication Technology, pp.529-534, 2013.

[35] P. Mayberry, "Increasing Pharmaceutical Compliance through Better Packaging," Annual National Symposium on Patient Compliance, 2009.

[36] J. Gao, Z. Pang, Q. Chen, and L.-R. Zheng, "Interactive packaging solutions based on RFID technology and Controlled Delamination Material," IEEE International Conference on RFID, pp.158-165, Apr 2010.

[37] Whitepaper - EPCglobal Class 1 Gen 2 RFID Specification, Alien Technology Corporation, 2005.

[38] Z. Pang, Q. Chen, and L. Zheng, "A pervasive and preventive healthcare solution for medication noncompliance and daily monitoring," 2nd International Symposium on Applied Sciences in Biomedical and Communication Technologies, pp. 1-6, Nov. 2009.

[39] Y.-M. Chi, T.-P. Jung, and G. Cauwenberghs, "Dry-Contact and Noncontact Biopotential Electrodes: Methodological Review," IEEE Reviews in Biomedical Engineering, vol.3, pp.106-119, 2010.

[40] J. Yoo, Y. Long, L. Seulki, H. Kim, and H.-J. Yoo, "A Wearable ECG Acquisition System With Compact Planar-Fashionable Circuit Board-Based Shirt," IEEE Transactions on Information Technology in Biomedicine, vol.13, no.6, pp.897-902, Nov. 2009.

[41] Z. Pang, L. Zheng, J. Tian, S. Kao-Walter, E. Dubrova, and Q. Chen, "Design of a terminal solution for integration of in-home health care devices and services towards the Internet-of-Things," Enterprise Information Systems, vol.7, pp.1-31, Apr. 2013.

[42] D. Buss, et al., "SOC CMOS technology for personal Internet products," IEEE Transactions on Electron Device, vol.50, no.3, pp. 546- 556, Mar 2003

[43] G. Yang, J. Chen, F. Jonsson, H. Tenhunen, and L-R. Zheng, "A Multi-Parameter Bio-Electric ASIC Sensor with Integrated 2-Wire Data Transmission Protocol for Wearable Healthcare System," Design, Automation \& Test in Europe, Mar. 2012.

[44] T.-C. Huang, and K.-T. Cheng, "Design for Low Power and Reliable Flexible Electronics: Self-Tunable Cell-Library Design," Journal of Display Technology, vol.5, no.6, pp.206-215, Jun. 2009.
[45] A. Nathan, et al.. "Flexible Electronics: The Next Ubiquitous Platform," Proceedings of the IEEE, vol.100, no. Special Centennial Issue, pp.1486-1517, May 2012.

[46] Y. Huang, J. Chen, Z. Yin, and Y. Xiong, "Roll-to-Roll Processing of Flexible Heterogeneous Electronics With Low Interfacial Residual Stress," IEEE Transactions on Components, Packaging and Manufacturing Technology, vol.1, no.9, pp.1368-1377, Sept. 2011.

[47] K. Hecker. (2009). OE-A Roadmap for Organic and Printed Electronics, (3rd ed.), VDMA Verlag GmbH.

[48] M. Singh, H. M. Haverinen, P. Dhagat, and G. E. Jabbour, "Inkjet Printing_Process and Its Applications," Advanced Materials, vol. 22, no. 6, pp. 673-685. Feb. 2010.

[49] V. Pekkanen, et al., "Utilizing inkjet printing to fabricate electrical interconnections in a System-in-Package," ELSEVIER Microelectronic Engineering, vol. 87, no. 11, pp. 2382-2390. Nov. 2010.

[50] L. Xie, Y. Feng, M. Mantysalo, Q. Chen, and L.-R. Zheng, "Integration of f-MWCNT Sensor and Printed Circuits on Paper Substrate," IEEE Sensors Journal, vol.13, no.10, pp.3948-3956, Oct. 2013.

[51] G. Yang, L. Xie, M. Mantysalo, J. Chen, T. Hannu, and L.-R. Zheng, "Bio-Patch Design and Implementation Based on a Low-Power System-on-Chip and Paper-based Inkjet Printing Technology," IEEE Transactions on Information Technology in Biomedicine, vol.16, no.6, pp.1043-1050, Nov. 2012.

[52] G. Yang, J. Chen, L. Xie, J. Mao, T. Hannu, and L.-R. Zheng, "A Hybrid Low Power Bio-Patch for Body Surface Potential Measurement," IEEE Journal of Biomedical and Health Informatics, vol.17, no.3, pp.591-599, May 2013.

[53] L. Xie, G. Yang, M. Mantysalo, L.-L. Xu, F. Jonsson, and L.-R. Zheng, "Heterogeneous Integration of Bio-Sensing System-on-Chip and Printed Electronics," IEEE Journal on Emerging and Selected Topics in Circuits and Systems, vol.2, no.4, pp.672-682, Dec. 2012.

[54] L. Li, and W. Benton, "Hospital technology and nurse staffing management decisions," Journal of Operations Management, vol.24, no.5, pp.676-691, 2006.

[55] L. Li, and C. Markowski, "An analysis of hospital capacity management patterns using Miles and Snow Topology," International Journal of Management and Enterprise Development, vol.3, no.4, pp.312-338, 2006.

[56] $\mathrm{L}$. $\mathrm{Li}$, "Technology designed to combat fakes in the global supply chain," Business Horizons, vol.56, no.2, pp.167-177, 2013.

[57] S. Fang, L. Xu, H. Pei, and Y. Liu, "An Integrated Approach to Snowmelt Flood Forecasting in Water Resource Management," IEEE Transactions on Industrial Informatics, vol.10, no.1, pp.548-558, 2014.

[58] L. Xu, "Introduction: Systems Science in Industrial Sectors," Systems Research and Behavioral Science, vol.30, no.3, pp.211-213, 2013.

[59] L. Xu, "Information Architecture for Supply Chain Quality Management," International Journal of Production Research, vol.49, no.1, pp.183-198, 2011.

[60] L. Xu, W. He, and S. Li, "Internet of Things in Industries: A Survey," IEEE Transactions on Industrial Informatics, 2014, DOI: 10.1109/TII.2014.2300753.

[61] B. M. Wilamowski, and O. Kaynak, "Oil well diagnosis by sensing terminal characteristics of the induction motor," IEEE Transactions on Industrial Electronics, vol.47, no.5, pp.1100-1107, 2000.

[62] M. Kataev, L. Bulysheva, A. Emelyanenko, and V. Emelyanenko, "Enterprise systems in Russia: 1992-2012," Enterprise Information Systems, vol.7, no.2, pp.169-186, 2013

[63] X. Chen, A. Guan, X. Qiu, H. Huang, J. Liu, and H. Duan, "Data configuration in railway signaling engineering-an application of enterprise systems techniques," Enterprise Information Systems, vol.7, no.3, pp.354-374, 2013.

[64] X. Wang, and X. Xu, "DIMP: an interoperable solution for software integration and product data exchange," Enterprise Information Systems, vol.6, no.3, pp.291-314, 2012.

[65] X. Chen, and Y. Fang, "Enterprise systems in financial sector-an application in precious metal trading forecasting," Enterprise Information Systems, vol.7, no.4, pp.558-568, 2013.

[66] C. Le, X. Gu, K. Pan, F. Dai, and G. Qi, "Public and expert collaborative evaluation model and algorithm for enterprise knowledge," Enterprise Information Systems, vol.7, no.3, pp.375-393, 2013.

[67] N. Li, W. Yi, Z. Bi, H. Kong, and G. Gong, "An optimization method for complex product design," Enterprise Information Systems, vol.7, no.4, pp.470-489, 2013 
[68] L. Li, and J. Liu, "An efficient and flexible web services-based multidisciplinary design optimization framework for complex engineering systems," Enterprise Information Systems, vol.6, no.3, pp.345-371, 2012

[69] W. Tan, W. Xu, F. Yang, L. Xu, and C. Jiang, "A framework for service enterprise workflow simulation with multi-agents cooperation," Enterprise Information Systems, vol.7, no.4, pp.523-542, 2013.

[70] H. Yu, T. Xie, S. Paszczynski, and B. M. Wilamowski, "Advantages of Radial Basis Function Networks for Dynamic System Design," IEEE Transactions on Industrial Electronics, vol. 58, no.12, pp.5438-5450, 2011

[71] F. Wang, B. Ge, L. Zhang, Y. Chen, Y. Xin, and X. Li, "A system framework of security management in enterprise systems," Systems Research and Behavioral Science, vol.30, no.3, pp.287-299, 2013.

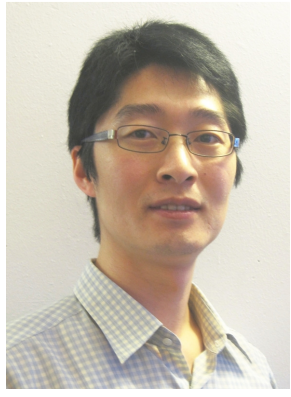

Dr. Geng Yang received his M.S. degree from the College of Biomedical Engineering and Instrument Science, Zhejiang University, Hangzhou, China, in 2006, and Ph.D. degree from Electronic Systems from the Royal Institute of Technology (KTH), Stockholm, Sweden, in 2013. He is currently working as a Post-Doc researcher in iPack VINN Excellence Center, the School of Information and Communication Technology (ICT), KTH, Stockholm, Sweden. He developed low power, low noise bio-electric $\mathrm{SoC}$ sensors for m-health. His research interests include flexible and stretchable electronics, mixed-mode IC design, low-power biomedical microsystem, wearable bio-devices, intelligent sensors and Internet-of-Things for healthcare.

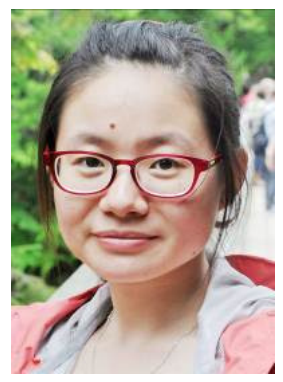

Li Xie received the B.Eng. degree in Electronic Information Engineering from Beihang University, Beijing, China, in 2003, and the M.S. degree in Information and Communication from Beijing Institute of Technology, Beijing, China, in 2009. She is currently working toward the Ph.D. degree in iPack VINN Excellence Center, the School of Information and Communication Technology (ICT), KTH, Stockholm, Sweden. Her main interests include printed electronics, flexible electronics, and heterogeneous integration for electronic systems.

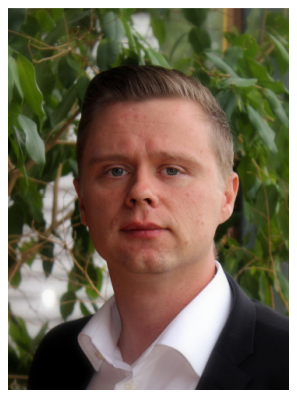

Dr. Matti Mäntysalo received his M.Sc. and D.Sc.(Tech) degrees in Electrical Engineering Tampere University of Technology, Tampere, Finland in 2004 and 2008, respectively.

$\mathrm{He}$ is an Asst. Prof. in Electronics materials and manufacturing and Adj. Prof. in Digital fabrication at TUT. Mäntysalo has led the Printable Electronics research group at TUT since 2008. He was a visiting scientist in iPack VINN Excellence Center, School of information and Communication Technology, KTH Royal Institute of Technology, Stockholm, Sweden, from 2011 to 2012. His research interests include printed electronics materials, fabrication processes, pre- and post-treatments, quality and performance analyses, reliability and failure analyses, and especially integration of printed electronics with silicon-based technology.

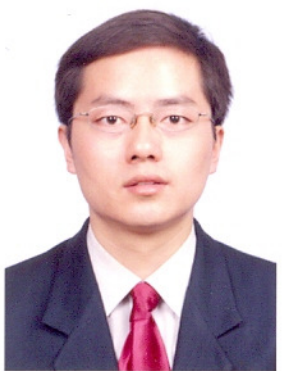

Dr. Xiaolin Zhou (M'07) received the B.Eng. degree in Microelectronics Engineering from Xidian University, Xi' an, China, in 1996, the M.S. degree in Communications and Information Systems from Nanjing University of Aeronautics and Astronautics, Nanjing, China, in 1999, and the $\mathrm{Ph} . \mathrm{D}$. degree in Communications and Information Systems from Shanghai Jiao-Tong University, China, in 2003.

$\mathrm{He}$ has been with the Department of Communication Science and Engineering, Fudan University, since 2007, where he is currently an Associate Professor. His research interests include wireless network technologies and intelligent medical instrumentation.

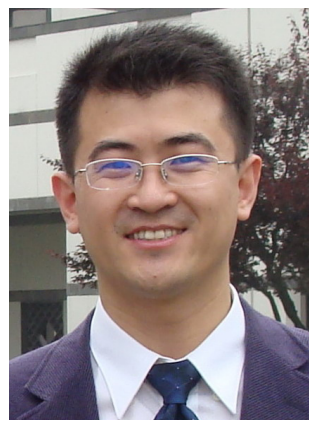

Dr. Zhibo Pang received B.Eng. degree in Electronic Engineering from Zhejiang University, Hangzhou, China, in 2002, MBA in Innovation and Growth from University of Turku, Turku, Finland, in 2012, and Ph.D. degree in Electronic and Computer Systems from the Royal Institute of Technology (KTH), Stockholm, Sweden, in 2013.

$\mathrm{He}$ is currently a research scientist at ABB Corporate Research, Västerås, Sweden. Before joined ABB, he worked as technical manager in semiconductor industry, designing baseband and application processors and turn-key solutions for mobile smart devices. He has 15 patents and over 30 peer-reviewed papers in international journals and conferences. He was awarded the National Great Invention Award by the Ministry of Information Industry of China in 2005, won the First Place Prize of the RFID Nordic EXPO in 2008 and Outstanding Paper Awards in ICACT2013. His current research interests include the Internet-of-Things, wireless sensor network, industrial communication, real time embedded system, enterprise information systems, automation networks, and multicore system-on-chip and network-on-chip. He also works on the business-technology joint research such as business model design, value chain formulation, strategy, and entrepreneurship \& intrapreneurship.

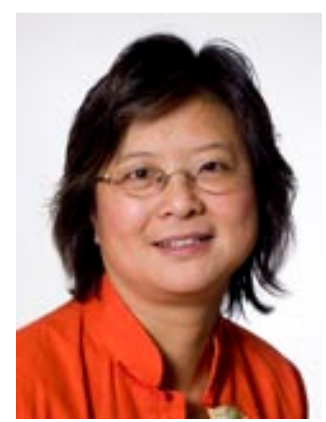

Dr. Sharon Kao-Walter received B.Eng. degree in Shanghai Jiao-Tong University. After staying in Lund Institute of Technology and Tetra Pak, she was back to university 1999 and received the Ph.D. degree in Blekinge Institute of technology in 2005.

Currently, she is working as Associate Professor at the Department of Mechanical Engineering in Blekinge Institute of Technology. She is also a visiting professor in Kunming University of Technology and Oversea Scholar at Shanghai Second Polytechnic University. Her main research focus is the application of Solid Mechanics and Mechanical Engineering in Packaging and Healthcare.

Dr. Qiang Chen received the Ph.D. degree in Electronics from Linköping University, Linköping, Sweden, in 1993. He was with Ericsson Microelectronics, Stockholm, Sweden, from 1994 to 2002 and became an Ericsson Corporate Expert in 2001. He joined Infineon Technologies Nordic, Stockholm, in 2003, as a Principal. He has been with the Royal Institute of Technology (KTH), Stockholm, since 2008, as a Senior Research Fellow and project leader with the Department of Electronic Systems and the iPack VINN Excellence Center, School of Information and Communication Technology. 
He has authored and co-authored more than 100 peer-reviewed scientific papers. His current research experiences and interests include RF electronic devices and circuits, wireless sensor network and sensor technology, Internet-of-Things (IoT) and printed electronics.

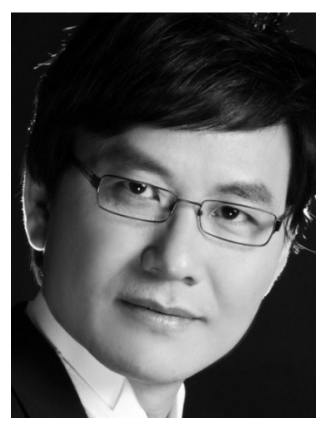

Dr. Li-Rong Zheng (M'01) is a chair professor in media electronics at KTH the Swedish Royal Institute of Technology, founder and director of iPack VINN Excellence Center at KTH. Since 2010, he holds the position as a distinguished professor and dean of ICT School at Fudan University, Shanghai, China.

He received his Ph.D. degree in electronic system design from $\mathrm{KTH}$, Stockholm Sweden in 2001. Since then, he was with KTH as a research fellow and became docent in 2003. He becomes full professor at KTH since 2006, director of iPack VINN Excellence Center and Ericsson Expert in 2007. His research experience and interest includes electronic circuits and systems for ambient intelligence and media applications, wireless sensors and internet-of-things for mobile Healthcare, intelligent logistics and food safety etc. He was involved in several strategic/expert groups both in EU and in China, particularly in areas of large area electronics, RFID and Internet-of-Things. He has authored and co-authored over 300 international reviewed publications and over 30 invited talks. He serves in the steering board of International Conference on Internet-of-Things (IoT) and the general chair of IoT2012, TPC members of several IEEE Conferences including ESSCIRC, ESSDERC, RFID, ESTC and EPTC etc. 